\title{
Universiteit
}

Leiden

The Netherlands

\section{Probing redox proteins on a gold surface by single molecule fluorescence spectroscopy}

Elmalk, A.T.; Salverda, J.M.; Tabares, L.C.; Canters, G.W.; Aartsma, T.J.

\section{Citation}

Elmalk, A. T., Salverda, J. M., Tabares, L. C., Canters, G. W., \& Aartsma, T. J. (2012). Probing redox proteins on a gold surface by single molecule fluorescence spectroscopy. Journal of Chemical Physics, 136, 235101. doi:10.1063/1.4728107

Version: $\quad$ Not Applicable (or Unknown)

License: $\quad$ Leiden University Non-exclusive license

Downloaded from: https://hdl.handle.net/1887/72401

Note: To cite this publication please use the final published version (if applicable). 


\section{Probing redox proteins on a gold surface by single molecule fluorescence spectroscopy}

Cite as: J. Chem. Phys. 136, 235101 (2012); https://doi.org/10.1063/1.4728107

Submitted: 09 January 2012 . Accepted: 24 May 2012 . Published Online: 15 June 2012

Abdalmohsen T. Elmalk, Jante M. Salverda, Leandro C. Tabares, Gerard W. Canters, and Thijs J. Aartsma

ARTICLES YOU MAY BE INTERESTED IN

Surface plasmon broadening for arbitrary shape nanoparticles: A geometrical probability approach

The Journal of Chemical Physics 119, 3926 (2003); https://doi.org/10.1063/1.1587686

Quantifying fluorescence enhancement for slowly diffusing single molecules in plasmonic near fields

The Journal of Chemical Physics 148, 123334 (2018); https://doi.org/10.1063/1.5023171

The Journal 


\title{
Probing redox proteins on a gold surface by single molecule fluorescence spectroscopy
}

\author{
Abdalmohsen T. Elmalk, Jante M. Salverda, Leandro C. Tabares, ${ }^{a)}$ Gerard W. Canters, ${ }^{\text {b) }}$ \\ and Thijs J. Aartsma \\ Leiden Institute of Physics, Leiden University, Huygens Laboratory, Niels Bohrweg 2, 2333CA Leiden, \\ The Netherlands
}

(Received 9 January 2012; accepted 24 May 2012; published online 15 June 2012)

\begin{abstract}
The interaction between the fluorescently labeled redox protein, azurin, and a thin gold film is characterized using single-molecule fluorescence intensity and lifetime measurements. Fluorescence quenching starts at distances below $2.3 \mathrm{~nm}$ from the gold surface. At shorter distances the quantum yield may decrease down to fourfold for direct attachment of the protein to bare gold. Outside of the quenching range, up to fivefold enhancement of the fluorescence is observed on average with increasing roughness of the gold layer. Fluorescence-detected redox activity of individual azurin molecules, with a lifetime switching ratio of 0.4 , is demonstrated for the first time close to a gold surface. (C) 2012 American Institute of Physics. [http://dx.doi.org/10.1063/1.4728107]
\end{abstract}

\section{INTRODUCTION}

Redox reactions drive a plethora of biological and chemical processes ranging from photosynthesis and respiration to industrial catalysis and the operation of fuel cells. ${ }^{1,2}$ Recently, due to their unique properties redox proteins have gained strong interest because of possible applications in biomolecular electronics and biosensing. However, to manipulate single redox proteins and attach them to an electrode surface without affecting their biochemical activity is a challenge. Gold has been used extensively for this purpose as it has a higher surface stability than other metals or non-metallic electrodes, while the surface chemistry of gold is well understood. ${ }^{3-5}$

To monitor the activity of redox proteins under singlemolecule conditions, it is necessary to maximize sensitivity and specificity. Our recent research efforts have targeted the implementation of a novel fluorescence method allowing single-molecule observation of redox events with enhanced sensitivity. ${ }^{6-9}$ The method is based on the fluorescent labeling of oxido-reductases. Optical tracking of electrochemical events made it possible to determine the electrochemical parameters and redox activity of ensembles of as few as 100 protein molecules. ${ }^{10-12}$

For the combination of fluorescent redox state detection and electronic control the protein together with its fluorescent label needs to be placed close to an electrode, usually a metal surface. This raises questions about the fluorophoremetal interaction. The subject has drawn considerable interest in recent years because of fundamental and applicationoriented reasons. ${ }^{13}$ The effect of a metal surface on a nearby fluorophore can lead to both enhancement and quenching of the fluorescence. ${ }^{14-24}$

\footnotetext{
a) Present address: CEA, Institut de Biologie et Technologies de Saclay, Service de Bioénergétique, Biologie Structurale et Mécanismes, CNRS URA 2096, 91191 Gif-sur-Yvette, France.

b) Author to whom correspondence should be addressed. Electronic mail: canters@ chem.leidenuniv.nl. Tel.: +31-71-527 4256. Fax: +31-71-527 4349 .
}

A striking aspect of the fluorophore-metal interaction is its strong and complicated distance dependence. At values of $d$ (where $d$ is the distance between the fluorophore and the metal surface) that are of the order of the wavelength of the exciting light $(d>500 \mathrm{~nm})$, interference occurs (both positive and negative) between light emitted by the fluorophore that reaches the observer directly and light emitted by the fluorophore that reaches the observer after being reflected off the metal surface. ${ }^{24-26}$ At intermediate ranges $(10 \mathrm{~nm}<d<500 \mathrm{~nm}$ ), the interaction of the molecular transition dipole with the nearby metal surface plasmons can lead to fluorescence quenching as well as to enhanced emission and excitation rates. ${ }^{18,20,26}$ At very close ranges $(d<10 \mathrm{~nm})$ energy transfer from the oscillating molecular transition dipole to the free conduction electrons of the metal may occur, also denoted as electron-hole energy transfer. ${ }^{27,28}$ On theoretical grounds it has been stated that the efficiency of the latter mechanism will be proportional to $1 / d^{3}$ or $1 / d^{4}$ depending on the exact value of $d$ and on the metal. ${ }^{18,24,26,29}$ Since the early work of Drexhage and co-workers ${ }^{25,30}$ numerous studies have appeared on fluorescence enhancement and quenching in relation to the distance between fluorophore and metal surface. ${ }^{17-19,21,24-26,28,30-36}$ However, the short distance regime $(d<\sim 2 \mathrm{~nm})$ has received relatively little attention. Still, even at these very small distances enhancement of the excitation rate can occur, especially for rough metal surfaces. This is the regime that we focus on.

We studied the fluorescence of labeled redox protein molecules immobilized on silanized glass or on gold films (bare or coated with a self-assembling monolayer (SAM)) as a function of the thickness of the gold film and the thickness of the SAM. Intensity and lifetime measurements allowed for the separation of enhancement and quenching effects on the fluorescence. In addition, the effect of the redox state on the fluorescence of the labeled protein was investigated. While this effect has been successfully studied for the enzyme nitrite reductase (NiR) at the single molecule level, ${ }^{7,9}$ 
the influence of a nearby Au layer has not been reported on so far.

The results of the present study are of relevance for the implementation of redox proteins in bio-optoelectronic (nano)devices. To the best of our knowledge, this study is the first successful attempt to examine the single molecule fluorescence lifetimes of labeled redox proteins immobilized on a metal surface.

\section{EXPERIMENTAL METHODS}

\section{A. Protein labeling}

Azurin from Pseudomonas aeruginosa was used as a redox protein model. It is a small $\left(M_{\mathrm{r}}=14.6 \mathrm{kDa}\right)$ blue copper protein that functions as an electron carrier possibly in the oxidative stress response of the organism. ${ }^{37}$ The intense $600 \mathrm{~nm}$ absorption band of this protein in the oxidized state is absent in the reduced state, which makes it suitable for redox state detection by fluorescent labeling. When labeling the protein covalently with a fluorescent label, the redox co-factor of the azurin (i.e., the $\mathrm{Cu}$ center, which itself is non-fluorescent) and the label may form a FRET pair by which a change in redox state is reflected by a change in fluorescence intensity and fluorescence lifetime of the label. ${ }^{7-9,38,39}$

Preparation and purification of the K27C azurin variant was done as previously described. ${ }^{38}$ The protein was labeled by incubation of a solution of $0.5 \mathrm{mM}$ protein in $20 \mathrm{mM}$ Hepes, $p \mathrm{H}$ 8.3, with a 5 times molar excess of Atto-655 succinimidyl ester (ATTO-TEC GmbH, Germany) for $1 \mathrm{~h}$ at room temperature for $\mathrm{N}$-terminal labeling. The unbound dye was removed using centrispin-10 columns (Princeton Separations; Adelphia, NJ, USA). Labeled protein was diluted with

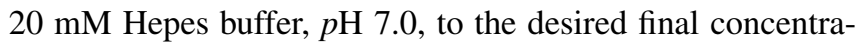
tions as determined by UV-vis spectroscopy.

\section{B. Preparation of surfaces}

All glass slides (Menzel, Germany) used to prepare gold films and silanized glass were sonicated in spectrometer grade acetone (30 $\mathrm{min}$ ), washed several times with water (Millipore water), dipped in $10 \% \mathrm{NaOH} / \mathrm{H}_{2} \mathrm{O}$ (30 min), washed again several times with water and stored in methanol. Before use the cover slips were dried and ozone-cleaned (UVP PR-100 UV-ozone photoreactor) for $1 \mathrm{~h}$ immediately before silanization or sputtering.

Before the preparation of gold films, first a $1 \mathrm{~nm}$ thick adhesion layer of molybdenum-germanium (MoGe) film was prepared by depositing MoGe onto freshly cleaned glass slides by magnetron sputtering (ATC 1800-F system (AJA corporation); deposition rate: $1.32 \mathrm{~nm} / \mathrm{min}$ in a $10 \mathrm{mTorr}$ Argon environment). Subsequently, gold films of specified thickness were prepared by sputtering at a deposition rate of $9.06 \mathrm{~nm} / \mathrm{min}$ (10 mTorr environment composed of a mixture of argon with $1 \%$ oxygen) on top of the MoGe film. The $\mathrm{Au}$ films were used immediately after preparation. Their thickness was varied between 10 and $100 \mathrm{~nm}$.

\section{Atomic force microscopy}

Atomic force microscopy (AFM) imaging of the samples was performed with a commercial AFM microscope (Nanoscope IIIa, Veeco, USA). Tapping mode images in air were acquired with an E-scanner (14 $\mu \mathrm{m}$ range), using $\mathrm{Si}$ probes with a resonance frequency of $75 \mathrm{kHz}$ and a nominal spring constant of $2.8 \mathrm{Nm}^{-1}$.

\section{Protein immobilization}

In one type of experiment the labeled protein was immobilized on a glass slide or a sputtered bare gold surface for optical measurements. Immobilization on glass was achieved by first depositing a layer of a 100:1 mixture of triethoxysilane (TES) and mercaptopropyl trimethoxysilane (MPTS) on a cleaned glass slide (chemicals from Fluka, used as received). The K27C azurin was bound to the silanized glass through a 1-11-bismaleimidotetraethyleneglycol linker $\left(\mathrm{BM}(\mathrm{PEO})_{3}\right.$, Pierce) as described by Kuznetsova et al. ${ }^{7}$ Immobilization on bare gold was realized by incubating the Au film with a solution of the $\mathrm{K} 27 \mathrm{C}$ azurin overnight at room temperature and rinsing the surface afterwards by flushing with buffer. This gave reproducible results of specifically immobilized K27C azurin without protein aggregation at the surface.

In a second type of experiment mixed SAMs of 1,nalkanedithiol $\mathrm{HS}\left(\mathrm{CH}_{2}\right)_{\mathrm{n}} \mathrm{SH}(n=4,6,8$, and 10) and $\mathrm{OH}-$ terminated alkanethiol $\mathrm{HS}\left(\mathrm{CH}_{2}\right)_{\mathrm{m}} \mathrm{OH}(m=n-2$, except for $n=4$ in which case $m=3$ ), denoted by Cnd ('d' denoting dithiol), were prepared by immersing slides with freshly sputtered $\mathrm{Au}$ films into a 2-propanol solution containing a mixture of $\mathrm{HS}\left(\mathrm{CH}_{2}\right)_{\mathrm{m}} \mathrm{OH}(10 \mathrm{mM})$ and $\mathrm{HS}\left(\mathrm{CH}_{2}\right)_{\mathrm{n}} \mathrm{SH}(1 \mathrm{mM})$ overnight at room temperature ${ }^{40}$ (all chemicals purchased from Aldrich Chemicals, dissolved in 2-propanol). The choice for $n>m$, i.e., for an alkanedithiol that is longer than the $\mathrm{OH}-$ terminated alkanethiol, was made to expose the reactive thiol of the linker. It provides the reaction site for immobilization of the $\mathrm{K} 27 \mathrm{C}$ azurin, while the $\mathrm{OH}$-terminated alkanethiol serves as the diluant to control the density of immobilized protein, as it prevents non-specific binding of K27C azurin (Fig. 1). Au slides were removed from solution, rinsed extensively with 2propanol, and dried in a pure $\mathrm{N}_{2}$ flow. A 200 pM solution of the labeled azurin (Az) in $20 \mathrm{mM}$ Hepes buffer, $p \mathrm{H} \mathrm{7,}$ was deposited onto the SAM-covered Au slide and left to incubate overnight at $4{ }^{\circ} \mathrm{C}$. The slide was then rinsed with $20 \mathrm{mM}$ Hepes buffer $(p \mathrm{H} 7)$ to remove free azurin. Measurements were performed in the same $20 \mathrm{mM}$ Hepes buffer $(p \mathrm{H} \mathrm{7})$ as used for immobilization.

In all experiments reduction was performed with sodium ascorbate, oxidation was performed by adding potassium ferricyanide. Reducing and oxidizing agents were added from freshly prepared stock solutions to final concentrations of $10 \mathrm{mM}$ sodium ascorbate or $1 \mathrm{mM}$ potassium ferricyanide.

The success of the immobilization procedure was verified using tapping mode AFM (Figures S1 and S2 in the supplementary material $^{41}$ ). For the mixed alkanedithiol/OHalkanethiol SAMs, distinct features with a height of $4.0 \pm 0.2 \mathrm{~nm}$ were observed. This value corresponds with the size of azurin $\left(3.5 \times 3.5 \times 4.4 \mathrm{~nm}^{3}\right)$ as determined by 


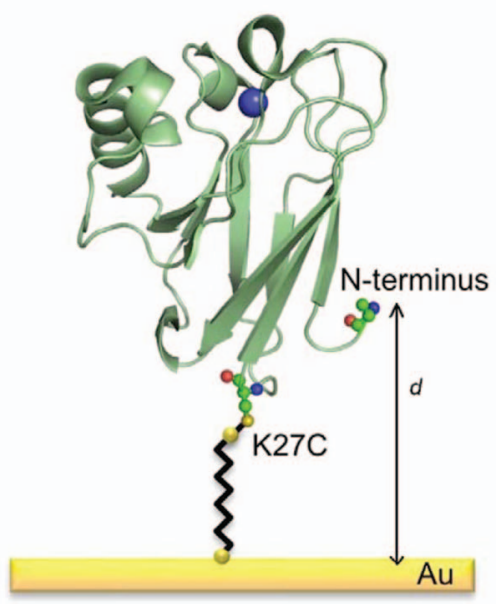

FIG. 1. Representation of the azurin (K27C) structure and schematic view of the covalent coupling of azurin to a SAM-modified Au surface. The distance between the dye label at the N-terminus and the Au surface is denoted by $d$.

x-ray crystallography. ${ }^{42}$ As a control for the specificity of the attachment on the SAM, azurin was incubated on a SAM of 8-mercapto-1-octanol only, applied on a $20 \mathrm{~nm}$ Au film. No protein was found on this surface after rinsing with pure buffer solution. The immobilization strategy described above, thus, establishes an effective and specific coupling between the protein and the gold surface.,, $43-45$

The change of surface morphology of the gold film with increasing thickness was also characterized by AFM. Calculated roughness values are given in Table I. Thin sputtered gold films $(10 \mathrm{~nm})$ have root-mean square (rms) height variations of about $0.2 \mathrm{~nm}$. With increasing thickness of the $\mathrm{Au}$ film, the roughness goes up, reaching $1.7 \mathrm{~nm} \mathrm{rms}$ value at $100 \mathrm{~nm}$ thickness. The thin Au films exhibit an exceptionally smooth and homogenous surface due to the presence of the MoGe wetting layer. At thicknesses above $20 \mathrm{~nm}$ the effect of the wetting layer diminishes and the surface roughness reaches values more typical of a sputtered metal surface.

\section{E. Confocal microscopy}

The fluorescence measurements were conducted on a home-built sample scanning confocal microscope. For fluorescence excitation a pulsed picosecond diode laser $(\lambda$ $=639 \mathrm{~nm}$ ) was used. Emission was detected at $\lambda=675 \mathrm{~nm}$ (a band pass filter with $50 \mathrm{~nm}$ spectral band width was employed in the detection path). Further details and details about the software used for data analysis are given in the supplemen-

TABLE I. Root-mean square (rms) values of height variations for various Au films, as determined by AFM.

\begin{tabular}{lc}
\hline \hline $\begin{array}{l}\text { Au thickness } \\
(\mathrm{nm})\end{array}$ & $\begin{array}{c}\mathrm{rms} \\
(\mathrm{nm})\end{array}$ \\
\hline 10 & 0.2 \\
20 & 0.3 \\
50 & 1.4 \\
100 & 1.7 \\
\hline \hline
\end{tabular}
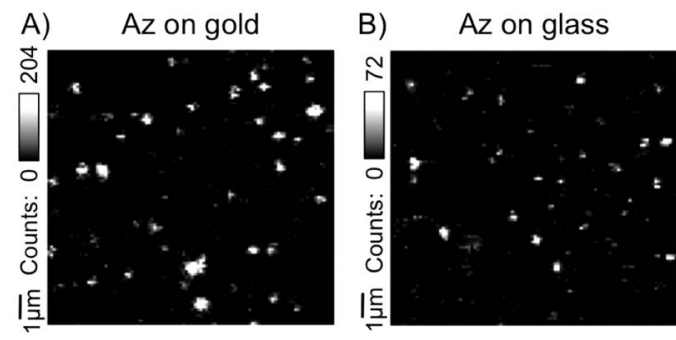

FIG. 2. $10 \times 10 \mu \mathrm{m}^{2}$ fluorescence images of immobilized reduced $\mathrm{K} 27 \mathrm{C}$ azurin (20 mM Hepes buffer, $p \mathrm{H} 7)$. A) on a $100 \mathrm{~nm}$ Au film coated with C10d SAM. B) on silanized glass (see the Experimental Methods). The scale bars show the intensity counts in $1.8 \mathrm{msec}$ bin time. The fluorescence intensities of individual spots on the $100 \mathrm{~nm}$ thick coated Au film are brighter than on glass (notice different scale bars).

tary material. ${ }^{41}$ Fluorescence intensity decays were analyzed as the sum of mono-exponential decays. ${ }^{46,47}$ (See supplementary material for further details ${ }^{41}$ ).

\section{RESULTS}

\section{A. Fluorescence intensity vs. film thickness}

To investigate the effect of Au film thickness on the fluorescence of labeled azurin, a single-molecule fluorescence experiment was carried out on labeled $\mathrm{K} 27 \mathrm{C} \mathrm{Az} \mathrm{immobilized} \mathrm{on}$ Au films of different thicknesses, coated with a C10d mixed SAM (no appreciable fluorescence quenching occurs for this SAM; vide infra). A characteristic confocal image (10 by $10 \mu \mathrm{m}$ ) of individual reduced azurin molecules on a $100 \mathrm{~nm}$ thick Au film coated with C10d SAM (in buffer) is shown in Fig. 2(a). For comparison, we also recorded a confocal fluorescence image of labeled azurin molecules on a glass surface, see Fig. 2(b). As can be seen from the scale bars in Fig. 2, there is a difference in fluorescence intensity of roughly a factor of 3-4.

In order to quantitatively compare the fluorescence properties for different azurin molecules and explore the underlying photophysics, we monitored the fluorescence of individual azurin molecules. A typical fluorescence time trace of a single, labeled reduced azurin molecule on a $100 \mathrm{~nm}$ thick Au film coated with a C10d mixed SAM is shown in Fig. 3, which corresponds to a fourfold higher fluorescence count rate than that of azurin on glass.

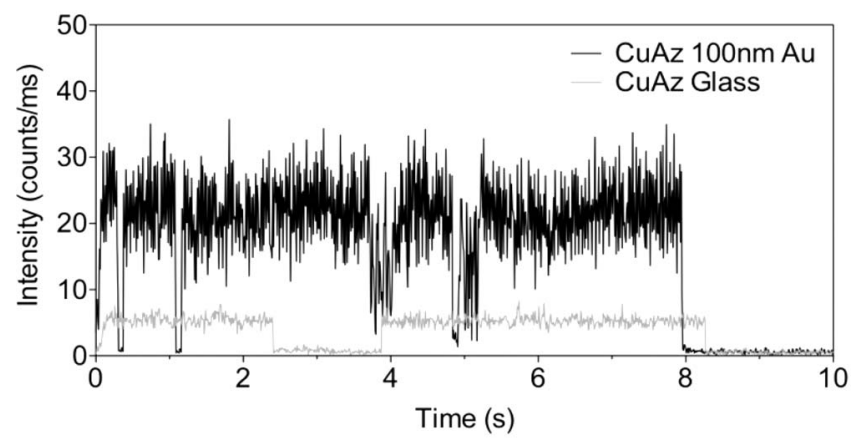

FIG. 3. Fluorescence time traces obtained from immobilized reduced $\mathrm{Cu}-\mathrm{Az}$ (K27C azurin) on $100 \mathrm{~nm}$ Au film coated with C10d SAM (black) and from reduced $\mathrm{Cu}-\mathrm{Az}$ on a silanized (see the Experimental Methods) glass surface (gray). 

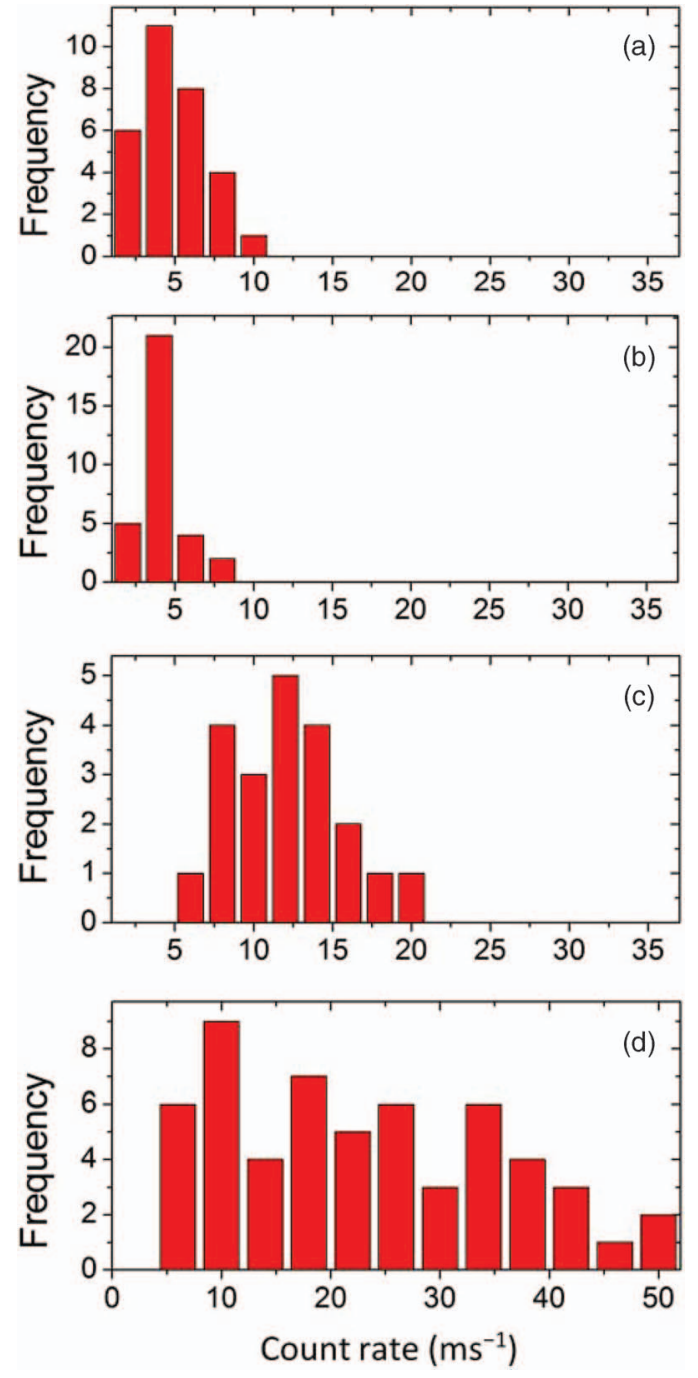

FIG. 4. Intensity count rate histograms for reduced K27C azurin molecules immobilized on silanized glass (A) and on Au films (B: $20 \mathrm{~nm}, \mathrm{C}: 50 \mathrm{~nm}$, and D: $100 \mathrm{~nm}$ thickness) coated with a C10d SAM.

In Fig. 4, count rate histograms of collections of individual molecules are shown for azurin on glass and on C10d coated Au films, the latter with thicknesses of $20 \mathrm{~nm}, 50$ $\mathrm{nm}$, and $100 \mathrm{~nm}$. All measurements were carried out at the same laser intensity of $1 \mathrm{~kW} / \mathrm{cm}^{2}$. The average count rate for reduced azurin on glass, reduced azurin on a C10d coated $10 \mathrm{~nm} \mathrm{Au}$ film (data not shown) and reduced azurin on a C10d coated $20 \mathrm{~nm}$ Au film are around 4 counts $/ \mathrm{ms}$. For the thicker C10d coated Au films (50 and $100 \mathrm{~nm}$ ) the average increases with film thickness $(12 \mathrm{c} / \mathrm{ms}$ and $22 \mathrm{c} / \mathrm{ms}$, respectively). At the same time the width of the intensity distribution increases considerably (from $2-8 \mathrm{c} / \mathrm{ms}$ to $6-20 \mathrm{c} / \mathrm{ms}$ and 6-50 c/ms, respectively). We think that the increase in average intensity with film thickness for the thicker films is due to the increase in surface roughness (see Table I), whereas the increase in the width of the intensity distribution reflects an increasing variation in surface topology.

In the protocol followed in this study the deposition of an $\mathrm{Au}$ film is preceded by the application of a thin, flat MoGe layer. In the subsequent $\mathrm{Au}$ sputtering step the MoGe layer promotes the growth of an equally flat Au layer but, appar-
TABLE II. Fluorescence lifetimes, $\tau$, of azurin immobilized on glass and on $50 \mathrm{~nm}$ Au films coated with SAMs of varying thicknesses.

\begin{tabular}{lc}
\hline \hline SAM & $\tau(\mathrm{ns})$ \\
\hline$\overline{\mathrm{a}}^{\mathrm{a}} \mathrm{d}$ & $0.7 \pm 0.1$ \\
C6d & $1.3 \pm 0.1$ \\
C8d & $2.3 \pm 0.1$ \\
C10d & $2.3 \pm 0.1$ \\
Glass & $2.5 \pm 0.1$ \\
\hline \hline
\end{tabular}

${ }^{a}$ Azurin immobilized directly on bare Au; no SAM.

ently, beyond 20-30 nm of Au the effect of the MoGe layer on the film flatness diminishes and the Au surface progressively becomes more uneven while also its topology becomes more varied. This is visible in the data shown in Fig. 4. It is worth noticing that the fluorescence enhancement seen when comparing the fluorescence image in Fig. 2(a) with the image in Fig. 2(b) is seen also in the intensity distributions presented in Fig. 4. Whereas the enhancement effect of the Au layer when compared with a glass substrate is minimal for a $10 \mathrm{~nm} \mathrm{Au}$ film, the average intensity increases 3- to 5-fold for the 50 and $100 \mathrm{~nm}$ films, respectively (see Fig. 4). ${ }^{48}$ In general, the fluorescence near metal surfaces is governed by a distancedependent competition between quenching and (local-field) enhancement. ${ }^{16,23}$ These aspects were examined in more detail by means of lifetime measurements.

\section{B. Fluorescence lifetime vs. SAM thickness}

When immobilizing proteins on a SAM-coated Au film the SAM layer forms a spacer between the gold surface and the protein. By using a series of $n$-alkanedithiols with lengths ranging from 4 to 10 carbon atoms the effect of a SAM thickness variation of from 5.9 to $12.5 \AA$ (Refs. 44, 49-51) on the fluorescence lifetime could be studied. The results are shown in Fig. 5 for a $50 \mathrm{~nm}$ thick Au film. Fluorescence decays were averaged over more than 500 single-molecule traces and quantified by fitting to mono-exponential decays convoluted with the instrument response function (IRF) (for lifetime distributions of individual molecules vide infra). Reduced azurin immobilized on C10d, the thickest SAM used, shows approximately the same lifetime as reduced azurin on glass (2.5 resp. $2.6 \mathrm{~ns})$. Significantly reduced lifetimes are observed for the shorter SAMs (C8d, C6d, and C4d; lifetimes of 2.3, 2.3, and $1.2 \mathrm{~ns}$, respectively), down to $0.7 \mathrm{~ns}$ for reduced azurin immobilized directly on $\mathrm{Au}$, see Table II.

To verify that the observed lifetimes were not affected by redox effects, the experiments were repeated with $\mathrm{Zn}$ azurin, which is redox-inactive. The lifetimes were similar to those observed for reduced $\mathrm{Cu}$-azurin. This confirms that the decrease in lifetime is not due to a redox-state dependent interaction between $\mathrm{Cu}$ site and dye label. ${ }^{39}$ We suggest that the reduced lifetime at shorter distances reflects the fluorescence quenching by molecule-metal interactions. Notably, labeled azurin molecules bound to C10d SAM seem to be at a distance which is sufficiently large to prevent fluorescence quenching, although the thickness of this SAM is only $1.3 \mathrm{~nm} .{ }^{49}$ 


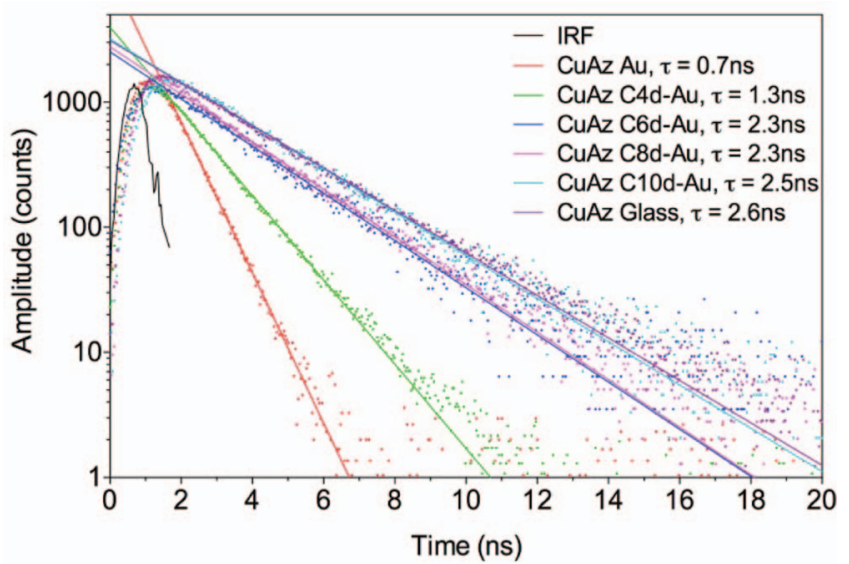

FIG. 5. Fluorescence decay curves of reduced azurin immobilized on silanized glass (purple), immobilized directly on $50 \mathrm{~nm} \mathrm{Au}$ film (red) and on $50 \mathrm{~nm} \mathrm{Au} \mathrm{films} \mathrm{coated} \mathrm{with} \mathrm{C4d} \mathrm{(green),} \mathrm{C6d} \mathrm{(blue),} \mathrm{C8d} \mathrm{(magenta),} \mathrm{or}$ C10d (cyan). IRF (black) is also included. Each decay curve represents an average over more than 500 individual molecules.

\section{Fluorescence lifetime vs. Au film thickness}

In order to examine the effect of the Au film thickness on the fluorescence lifetime, the measurement series described above was repeated for Au films with thicknesses of 10, 20, 50 , and $100 \mathrm{~nm}$. The fluorescence data were all collected under the same excitation and detection conditions, allowing a direct comparison between the different measurements (Fig. 6). The fluorescence lifetime appears to be independent of the Au film thickness.

The shortest fluorescence lifetimes were observed when azurin was adsorbed directly on the Au films, which is to be expected as quenching is known to be stronger at shorter distance. The lifetime of azurin bound to C10d is the longest and similar to the lifetime of azurin on glass. Thus, the fluorescence lifetime only depends on the distance between fluorophore and Au surface, independent of Au film thickness.

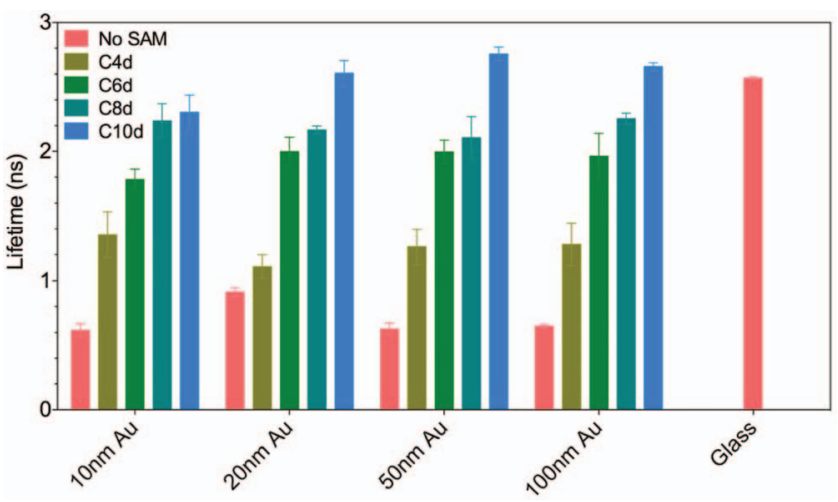

FIG. 6. Fluorescence lifetimes of reduced $\mathrm{Cu}-\mathrm{Az}$ immobilized on $\mathrm{Au}$ films of different film thicknesses (from 10 to $100 \mathrm{~nm}$ ) that are coated with C4d (olive), C6d (green), C8d (blue-green), and C10d (bright blue) SAMs and of reduced $\mathrm{Cu}-\mathrm{Az}$ adsorbed directly on $50 \mathrm{~nm} \mathrm{Au}$ (red). Also presented is the lifetime of reduced $\mathrm{Cu}-\mathrm{Az}$ immobilized on silanized glass (red). The lifetimes were obtained by monitoring the total emission of a $10 \times 10 \mu \mathrm{m}^{2}$ surface area. For each bar the lifetimes measured for a number of fluorescence images (varying from 2 to 16) were averaged. The error bars correspond to standard deviations.

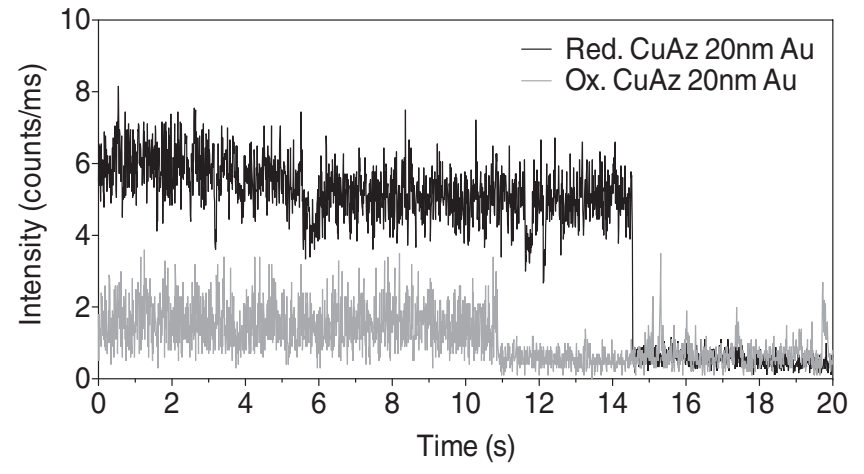

FIG. 7. Time traces for two individual labeled K27C azurin molecules immobilized on a $20 \mathrm{~nm}$ Au film coated with C10d SAM, one trace for azurin in the reduced state (black, $\tau_{\text {red }}=2.6 \mathrm{nsec}$ ), and one for azurin in the oxidized state (gray, $\tau_{\text {oxid }}=1.6 \mathrm{nsec}$ ). Data refer to two different samples. Reduction/oxidation was controlled by addition of $10 \mathrm{mM}$ ascorbate (reduced sample) or $1 \mathrm{mM}\left[\mathrm{Fe}(\mathrm{CN})_{6}\right]^{3-}$ (oxidized sample).

In conclusion it can be stated that, whereas the fluorescence lifetime is independent of Au film thickness, the fluorescence intensity does depend on it (see Fig. 4). Clearly, two different mechanisms play a role.

\section{Effect of redox state}

The experiments reported so far relate to azurin in the reduced state. To check how the redox state of the protein might affect the fluorescence behavior, we also measured the fluorescence intensity and lifetime for azurin in the oxidized state. The redox state could be easily controlled by the addition of reducing or oxidizing agents (see above). Figure 7 shows time traces for two individual azurin molecules immobilized on a $20 \mathrm{~nm}$ Au film coated with C10d SAM, one in the reduced state (black), and one in the oxidized state (gray). The intensity ratio between the two states is about a factor of 3 in this instance.

Lifetime histograms are shown in Fig. 8 for oxidized (bottom, left) and reduced (bottom, right) single azurin molecules bound to C10d SAM on $20 \mathrm{~nm}$ Au films, which appear similar to azurin on glass surfaces (top). Reduced and oxidized molecules exhibit a long ( $2.6 \pm 0.2 \mathrm{~ns}$, black bars) and a short lifetime ( $1.6 \pm 0.2 \mathrm{~ns}$, gray bars), respectively. The oxidized azurin lifetimes were found to be independent of the $\mathrm{Au}$ film thickness, just as for reduced azurin. For azurin immobilized on a C8d SAM on Au films of varying thicknesses, the reduced and oxidized forms exhibited lifetimes of 2.3 $\pm 0.2 \mathrm{~ns}$ and $1.1 \pm 0.2 \mathrm{~ns}$, respectively. Thus, it is still possible to discern the two redox states in the presence of fluorescence quenching by a metal surface. Lifetimes were also measured for oxidized azurin immobilized on C6d and C4d SAMs and on bare $\mathrm{Au}$. The results exhibit the same trend as the distance dependent fluorescence quenching data observed for reduced azurin.

\section{DISCUSSION}

In the following we discuss the distance dependence of the fluorescence quenching, the relation between fluorescence 


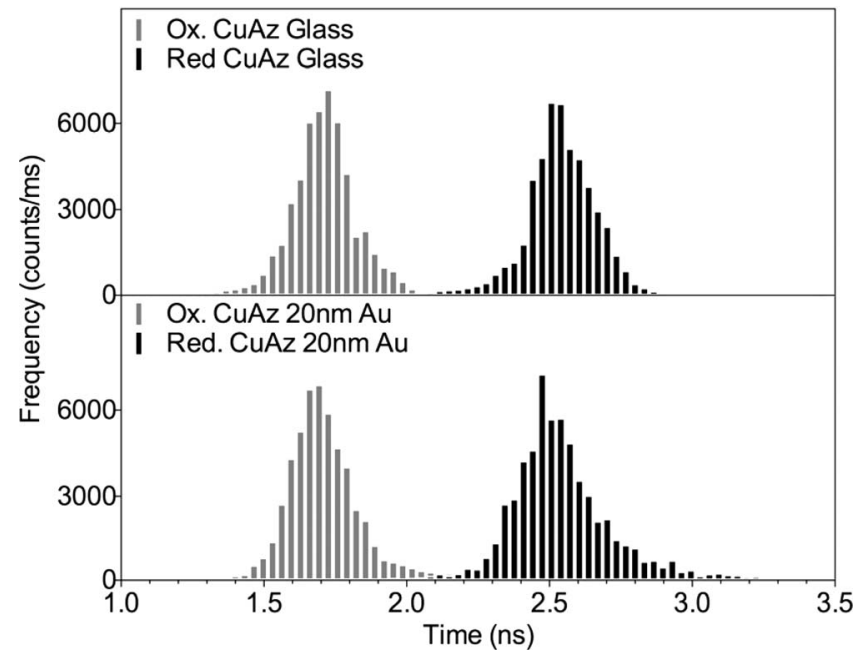

FIG. 8. Fluorescence lifetime histograms of labeled K27C azurin in the oxidized (grey histograms at the left) and the reduced state (black histograms at the right) immobilized on $20 \mathrm{~nm}$ Au coated with a C10d SAM (bottom) and immobilized on glass (top).

enhancement and surface roughness, and the redox switching behavior.

Both quenching and enhancing interactions between fluorophores and metal surfaces have an effect on the fluorescence intensity and lifetime, $\tau$. The fluorescence intensity (below saturation) is proportional to the fluorescence emission rate $\gamma_{\mathrm{em}}$, which is the product of the excitation rate $\gamma_{\mathrm{exc}}$ and quantum yield $q .{ }^{15}$ The radiative rate and the (intrinsic) nonradiative decay rate of the molecule will be denoted by $\gamma_{\mathrm{r}}$ and $\gamma_{\mathrm{nr}}$ and a zero subscript will be used to denote quantities for the molecule in free space. For a fluorophore in the absence of a metal surface one can write

$$
\begin{aligned}
& \gamma_{e m, 0}=\gamma_{e x c, 0} q_{0}=\gamma_{e x c, 0} \gamma_{r, 0} \tau_{0}, \\
& q_{0}=\left(\gamma_{r, 0} / \gamma_{r, 0}+\gamma_{n r, 0}\right)=\gamma_{r, 0} \tau_{0} .
\end{aligned}
$$

When the fluorophore is placed near the metal surface, both the excitation rate, $\gamma_{\text {exc }}$, and the emission rate, $\gamma_{\mathrm{r}}$, may be enhanced by the fluorophore-metal interaction, ${ }^{15,16,52}$ especially when dealing with rough surfaces. Moreover, at the very close ranges probed here (fluorophore-metal distance $d$ $<10 \mathrm{~nm}$ ) the interaction of the fluorophore with the free electrons in the metal may open up an additional radiationless decay channel (electron-hole energy transfer) characterized by a rate $\gamma_{\mathrm{abs}}{ }^{16}$ As a consequence, the expressions for $\gamma_{\mathrm{em}}$ and $q$ for a fluorophore near the metal can be written as ${ }^{18,24}$

$$
\begin{gathered}
\gamma_{e m}=\gamma_{e x c} q=\gamma_{e x c} \gamma_{r} \tau, \\
q=\left(\gamma_{r} / \gamma_{r}+\gamma_{n r, 0}+\gamma_{a b s}\right)=\gamma_{r} \tau,
\end{gathered}
$$

where we have assumed that the intramolecular radiationless decay rate is not affected by the presence of the metal film, i.e., $\gamma_{n r}=\gamma_{n r, 0}$.

We believe that $\gamma_{\mathrm{r}}$ is not affected in our measurements. This we conclude from the decoupling in our data between the lifetime changes (which are independent of $\mathrm{Au}$ film thickness and only occur for a change in SAM thickness) and the inten- sity enhancements (which do depend on Au film thickness). The lifetime is given by

$$
\tau=\frac{1}{\gamma_{r}+\gamma_{n r, 0}+\gamma_{a b s}},
$$

whereas the intensity is proportional to

$$
\gamma_{e m}=\frac{\gamma_{e x c} \gamma_{r}}{\gamma_{r}+\gamma_{n r, 0}+\gamma_{a b s}} \text {. }
$$

Thus, an increase in $\gamma_{\mathrm{r}}$ would affect both lifetime and fluorescence enhancement at the same time. Since this is not what is observed, we conclude that only $\gamma_{\text {abs }}$ and $\gamma_{\text {exc }}$ change and that $\gamma_{r} \approx \gamma_{r, 0}$. We now can check how the enhancement of $\gamma_{\mathrm{abs}}$ depends on distance. From the above approximate equality it follows that

$$
\begin{aligned}
& \tau_{0}=\frac{1}{\gamma_{r, 0}+\gamma_{n r, 0}}=\frac{1}{\gamma_{0}}, \\
& \tau=\frac{1}{\gamma_{r, 0}+\gamma_{n r, 0}+\gamma_{a b s}}=\frac{1}{\gamma_{0}+\gamma_{a b s}},
\end{aligned}
$$

and

$$
\frac{\gamma_{a b s}}{\gamma_{0}}=\frac{\tau_{0}}{\tau}-1,
$$

where $\gamma_{0}$ denotes the total decay rate, $\gamma_{0} \equiv \gamma_{r, 0}+\gamma_{n r, 0}$. The enhancement of $\gamma_{\text {abs }}$ as a function of the fluorophore metal distance calculated on the basis of Eq. (7) is presented in Table III. As can be seen in Fig. 9, the data conform to a $d^{-3}$ dependence of $\gamma_{\text {abs }} / \gamma_{0}$ (for a pictorial definition of $d$, see Fig. 1).

We briefly discuss the findings presented above.

The enhancement of excitation and emission by a metal film critically depend on the film roughness contrary to the enhancement of the radiationless damping of the fluorophore which mainly depends on the distance between emitter and metal surface. ${ }^{18,21,23,24,28,31,33,34,36,47,53-56}$ Fluorescence enhancement for dye molecules directly deposited on rough metallic surfaces has been reported before, also in the sub$5 \mathrm{~nm}$ regime. ${ }^{21,31,32,34,36,47,53-60}$ Within the accuracy of the experiment we find that the radiative rate is not enhanced (within an estimated accuracy of a factor of 1.5) while the excitation rate increases with increasing surface roughness by a factor of up to 3-4 (as measured for a $100 \mathrm{~nm}$ thick Au film). This increase of excitation rate is in line with the increase in

TABLE III. Distance dependence of the fluorescence lifetime $\tau$ and the metal energy transfer rate $\gamma_{\text {abs }}$.

\begin{tabular}{lccc}
\hline \hline SAM & $\mathrm{d} / \mathrm{nm}^{\mathrm{a}}$ & $\tau / \tau_{0} \mathrm{~b}$ & $\gamma_{\mathrm{abs}} / \gamma_{0}{ }^{\mathrm{c}}$ \\
\hline none & 1 & 0.25 & 3 \\
C4d & 1.6 & 0.46 & 1.2 \\
C6d & 1.8 & 0.77 & 0.3 \\
C8d & 2.0 & 0.77 & 0.3 \\
C10d & 2.3 & 1 & 0 \\
\hline \hline
\end{tabular}

$\overline{{ }^{a} \text { Distance from dye label to gold surface, calculated by increasing the SAM thickness }}$ by $1 \mathrm{~nm}$ to account for the distance between the label and the SAM layer (see Figure 1). SAM thickness was calculated according to Heering and Canters ${ }^{39}$ and Love et al. ${ }^{37}$ using a C-C bond length of $1.27 \AA$, a CS endgroup size of $1.79 \AA$ and a SAM/Au angle of $30^{\circ}$

${ }^{\mathrm{b}}$ Lifetime values taken from Table II; $\tau_{0}$ is taken from C10d data.

${ }^{\mathrm{c}}$ Calculated from Eq. (7). 


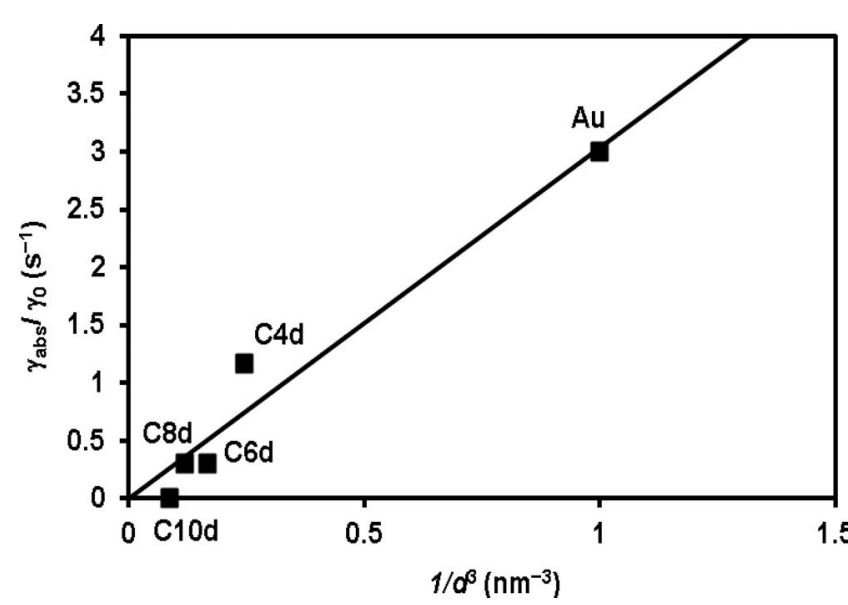

FIG. 9. Dependence of the energy transfer rate $\gamma_{\mathrm{abs}}$ on the inverse cubic distance, $1 / d .^{3}$ The straight line is a linear fit to the data points. Data taken from Table III.

surface roughness as established by AFM measurements (see Table I).

The discrepancy between excitation $\left(\gamma_{\text {exc }}\right)$ and emission $\left(\gamma_{\mathrm{r}}\right)$ enhancement at first sight may seem suspicious since on theoretical grounds reciprocity in the enhancement factors is predicted. ${ }^{16,26,36}$ However, the symmetry between the expressions for the two enhancement factors is not exact in the sense that the emission and excitation wavelengths are usually different. ${ }^{16,35,59,61,62}$ Since the enhancement is wavelength dependent, emission and excitation may be enhanced by different extents. Moreover, the dependence of the enhancement on dipole orientation of the emitter with respect to the metal surface and the polarization of the incoming light is different for emission and excitation. ${ }^{16,26,61,63}$ These effects may lead to a non-neglectable difference between the enhancement factors. The difference may become even more pronounced when the fluorophore is located in between two closely spaced metal particles that act as an optical antenna. ${ }^{36,61,64}$ Field enhancement factors up to $10^{4}-10^{5}$ have been predicted, depending on the metal, ${ }^{61,65}$ and experimental fluorescence enhancements factors of up to $10^{3}$ have been reported. ${ }^{61}$ It is conceivable that also on rough surfaces irregularities in the surface structure may lead to antenna-like configurations with similar enhancement effects ("hot spots"). ${ }^{62,65,66}$ Considering the modest fluorescence enhancement observed in the present study the contribution of possible hot spots, if any, to the fluorescence is not noticeable. Still, the lack of enhancement of the emission rate is remarkable and deserves further study.

Radiationless energy transfer from emitter to a metal surface has been shown for numerous cases to increase with decreasing distance between fluorophore and metal. For the distance regime considered in the present study, energy losses are supposed to derive from coupling of the molecular transition dipole moment with the Fermi gas of the free electrons in the metal also described as coupling with electron-hole pairs. ${ }^{34,36}$ When the coupling is restricted to surface electrons a $d^{-4}$ distance dependence has been predicted whereas a $d^{-3}$ dependence is expected for so-called volume energy transfer. ${ }^{18,24,28,35,36}$ For large distances volume energy transfer will prevail but the critical distance $d_{\mathrm{c}}$ where the $d^{-4}$ dependence changes into a $d^{-3}$ dependence varies with the metal. For instance for Pt and Ni $d_{\mathrm{c}} \approx 1 \AA$ whereas for $\operatorname{Ag} d_{\mathrm{c}} \approx 200 \AA{ }^{28}$ Our data (Fig. 9) favour a volume energy transfer mechanism.

The presence of two populations (enhanced and unenhanced emitters, both with the same lifetime, see Fig. 4) is ascribed to the microscopic features of the metal surface. It is well known that the electromagnetic field enhancement depends upon the local surface roughness and the surface morphology. ${ }^{54}$ Therefore, local variation in the surface morphology may lead to a variation in the enhancement.

The results on chemical oxidation and reduction of azurin show that the proteins remain redox active on the gold surfaces with the used immobilization strategy. To compare the redox switching as inferred from the intensity time traces and from the fluorescence lifetimes the amount of switching for both types of measurement was calculated. We find the intensity-based switching ratio, $Q_{\mathrm{I}}$, to be 0.7 with

$$
Q_{\mathrm{I}}=1-\left(F_{\text {oxid }} / F_{\text {red }}\right),
$$

in which $F_{\text {oxid }}$ and $F_{\text {red }}$ denote the fluorescence intensity of the $\mathrm{Az}$ in the reduced and oxidized form, respectively, ${ }^{39}$ while the lifetime-based switching ratio, ${ }^{67} Q_{\mathrm{L}}$,

$$
Q_{\mathrm{L}}=1-\left(\tau_{\text {oxid }} / \tau_{\text {red }}\right),
$$

appears to be 0.4 . Here, $\tau_{\text {oxid }}$ and $\tau_{\text {red }}$ denote the fluorescence lifetime of the $\mathrm{Az}$ in the reduced and oxidized form, respectively. The discrepancy between these two values is currently the subject of further investigation.

\section{CONCLUSION}

In summary, the combination of labeled azurin immobilized on Au with a mixed SAM has enabled us to characterize the label-metal interaction in detail. Fluorescence quenching is shown to be a short range effect, reflecting energy transfer between the fluorophore and conduction electrons of the metal. Significant fluorescence enhancement is observed with increasing roughness of the gold layer. These results are in good agreement with theoretical predictions based on earlier, non single-molecule, experiments with fluorophores on metal films.

The results also illustrate how the redox states of a single protein molecule can be investigated by fluorescence lifetime analysis both for Az on gold and for Az on glass. Thus, the combination of FLIM (fluorescence lifetime imaging) with our FRET-based redox detection method ${ }^{9}$ provides a new approach for studying the kinetics of biological electron transfer at the single molecule level. Furthermore, the ability to tune the emission properties of labeled redox proteins immobilized on metal surfaces opens a way to design improved biosensing devices.

\section{ACKNOWLEDGMENTS}

The authors thank Alessio Andreoni, MSc, for helpful suggestions. They are grateful to Professor M. Orrit for useful advice, to Dr. Thyra de Jong and Ing. Lionel Ndamba for assistance with protein purification, and to Dr. Razvan Stan and 
Mohammed Kamran, MSc, for assistance with AFM measurements. M.E. thanks the late Marten Durieux, the Stichting Steunfonds Soedanese Studenten, and Al Neelain University for supporting his stay in Leiden. J.M.S. was supported by a Veni Grant from NWO, the Netherlands Foundation for Scientific Research. L.C.T. was supported by the European Community through the EdRox Network (Contract No. MRTN-CT-2006-035649) and by NWO, the Netherlands Foundation for Scientific Research through a travel grant (Grant No. 040.11.223).

${ }^{1}$ I. Willner, Science 298, 2407 (2002).

${ }^{2}$ G. Gilardi and A. Fantuzzi, Trends Biotechnol. 19, 468 (2001).

${ }^{3}$ Q. J. Chi, O. Farver, and J. Ulstrup, Proc. Natl. Acad. Sci. U.S.A. 102, 16203 (2005)

${ }^{4}$ Y. Astier, G. W. Canters, J. J. Davis, H. A. O. Hill, M. P. Verbeet, and H. J. Wijma, ChemPhysChem 6, 1114 (2005).

${ }^{5}$ A. W. J. W. Tepper, J. Am. Chem. Soc. 132, 6550 (2010).

${ }^{6}$ R. H. Goldsmith, L. C. Tabares, D. Kostrz, C. Dennison, T. J. Aartsma, G. W. Canters, and W. E. Moerner, Proc. Natl. Acad. Sci. U.S.A. 108, 17269 (2011).

${ }^{7}$ S. Kuznetsova, G. Zauner, T. J. Aartsma, H. Engelkamp, N. Hatzakis, A. E. Rowan, R. J. M. Nolte, P. C. M. Christianen, and G. W. Canters, Proc. Natl. Acad. Sci. U.S.A. 105, 3250 (2008).

${ }^{8}$ R. Schmauder, F. Librizzi, G. W. Canters, T. Schmidt, and T. J. Aartsma, ChemPhysChem 6, 1381 (2005).

${ }^{9}$ L. C. Tabares, D. Kostrz, A. T. Elmalk, A. Andreoni, C. Dennison, T. J. Aartsma, and G. W. Canters, Chem.-Eur. J. 17, 12015 (2011).

${ }^{10}$ J. J. Davis, H. Burgess, G. Zauner, S. Kuznetsova, J. Salverda, T. Aartsma, and G. W. Canters, J. Phys. Chem. B 110, 20649 (2006).

${ }^{11}$ J. M. Salverda et al., Angew. Chem., Int. Ed. 49, 5776 (2010).

${ }^{12}$ L. Krzeminski, L. Ndamba, G. W. Canters, T. J. Aartsma, S. D. Evans, and L. J. C. Jeuken, J. Am. Chem. Soc. 133, 15085 (2011).

${ }^{13}$ R. Rinaldi et al., Appl. Phys. Lett. 82, 472 (2003).

${ }^{14}$ L. Andolfi et al., J. Electroanal. Chem. 565, 21 (2004).

${ }^{15}$ P. Anger, P. Bharadwaj, and L. Novotny, Phys. Rev. Lett. 96, 113002 (2006).

${ }^{16}$ P. Bharadwaj and L. Novotny, Opt. Express 15, 14266 (2007).

${ }^{17}$ R. R. Chance, A. Prock, and R. Silbey, J. Chem. Phys. 60, 2744 (1974).

${ }^{18}$ R. R. Chance, A. Prock, and R. Silbey, J. Chem. Phys. 62, 2245 (1975).

${ }^{19}$ R. R. Chance, A. Prock, and R. Silbey, J. Chem. Phys. 65, 2527 (1976).

${ }^{20}$ G. W. Ford and W. H. Weber, Phys. Rep., Phys. Lett. 113, 195 (1984).

${ }^{21}$ E. Fort and S. Gresillon, J. Phys. D 41, 013001 (2008).

${ }^{22}$ Y. Fu and J. R. Lakowicz, Laser Photonics Rev. 3, 221 (2009).

${ }^{23}$ J. R. Lakowicz, Anal. Biochem. 337, 171 (2005).

${ }^{24}$ D. H. Waldeck, A. P. Alivisatos, and C. B. Harris, Surf. Sci. 158, 103 (1985).

${ }^{25}$ K. H. Drexhage, Bull. Am. Phys. Soc. 14, 873 (1969).

${ }^{26}$ E. H. Hellen and D. Axelrod, J. Opt. Soc. Am. B 4, 337 (1987); K. H. Drexhage, J. Lumin. 12, 693 (1970).

${ }^{27}$ C. S. Yun, A. Javier, T. Jennings, M. Fisher, S. Hira, S. Peterson, B. Hopkins, N. O. Reich, and G. F. Strouse, J. Am. Chem. Soc. 127, 3115 (2005).

${ }^{28}$ B. N. J. Persson and N. D. Lang, Phys. Rev. B 26, 5409 (1982).

${ }^{29}$ L. Danos, R. Greef, and T. Markvart, Thin Solid Films 516, 7251 (2008).

${ }^{30}$ H. Bucher et al., Mol. Cryst. 2, 199-230 (1967).

${ }^{31}$ R. Aroca, G. J. Kovacs, C. A. Jennings, R. O. Loutfy, and P. S. Vincett, Langmuir 4, 518 (1988).

${ }^{32}$ F. R. Aussenegg, A. Leitner, M. E. Lippitsch, H. Reinisch, and M. Riegler, Surf. Sci. 189, 935 (1987)

${ }^{33}$ J. S. Biteen, D. Pacifici, N. S. Lewis, and H. A. Atwater, Nano Lett 5, 1768 (2005)
${ }^{34}$ E. Le Moal, S. Leveque-Fort, M. C. Potier, and E. Fort, Nanotechnology 20, 225502 (2009).

${ }^{35}$ A. Wokaun, H. P. Lutz, A. P. King, U. P. Wild, and R. R. Ernst, J. Chem. Phys. 79, 509 (1983).

${ }^{36}$ H. Metiu and P. Das, Annu. Rev. Phys. Chem. 35, 507 (1984).

${ }^{37}$ E. Vijgenboom, J. E. Busch, and G. W. Canters, Microbiology 143, 2853 (1997).

${ }^{38}$ S. Kuznetsova, G. Zauner, R. Schmauder, O. A. Mayboroda, A. M. Deelder, T. J. Aartsma, and G. W. Canters, Anal. Biochem. 350, 52 (2006).

${ }^{39}$ R. Schmauder, S. Alagaratnam, C. Chan, T. Schmidt, G. W. Canters, and T. J. Aartsma, JBIC, J. Biol. Inorg. Chem. 10, 683 (2005).

${ }^{40}$ X. D. Cui et al., Science 294, 571 (2001).

${ }^{41}$ See supplementary material at http://dx.doi.org/10.1063/1.4728107 for figures illustrating the features of azurin molecules on a C10d mixed SAM on gold and the difference in roughness between a $10 \mathrm{~nm}$ and a $100 \mathrm{~nm} \mathrm{Au}$ film.

${ }^{42}$ H. Nar, A. Messerschmidt, R. Huber, M. Vandekamp, and G. W. Canters, J. Mol. Biol. 221, 765 (1991).

${ }^{43}$ T. Wink, S. J. van Zuilen, A. Bult, and W. P. van Bennekom, Analyst 122, R43-R50 (1997).

${ }^{44}$ J. C. Love, L. A. Estroff, J. K. Kriebel, R. G. Nuzzo, and G. M. Whitesides, Chem. Rev. 105, 1103 (2005).

${ }^{45}$ D. Qu, B. C. Kim, C. W. J. Lee, and K. Uosaki, Bull. Korean Chem. Soc. 30, 2549 (2009)

${ }^{46}$ K. Ray, J. Zhang, and J. R. Lakowicz, Anal. Chem. 80, 7313 (2008).

${ }^{47}$ J. Zhang and J. R. Lakowicz, Opt. Express 15, 2598 (2007).

${ }^{48}$ As the penetration depth of light waves with a wavelength of around 630$650 \mathrm{~nm}$ into gold is small $(10-15 \mathrm{~nm})$ we do not expect the Au film thickness to play a significant role in the fluorescence quenching and enhancing mechanisms. See also the discussion (vide infra) on the distance dependence of the radiationless energy transfer and the references cited there.

${ }^{49}$ H. A. Heering and G. W. Canters in Engineering the Bioelectronic Interface: Applications to Analyte Biosensing and Protein Detection, edited by J. J. Davis (Royal Society of Chemistry, 2009), p. 119.

${ }^{50}$ C. O'Dwyer, G. Gay, B. V. de Lesegno, and J. Weiner, Langmuir 20, 8172 (2004).

${ }^{51}$ X. Yao, J. X. Wang, F. M. Zhou, J. Wang, and N. J. Tao, J. Phys. Chem. B 108, 7206 (2004).

${ }^{52}$ K. L. Kelly, E. Coronado, L. L. Zhao, and G. C. Schatz, J. Phys. Chem. B 107, 668 (2003).

${ }^{53}$ G. Chumanov, K. Sokolov, B. W. Gregory, and T. M. Cotton, J. Phys. Chem. 99, 9466 (1995).

${ }^{54}$ H. Shen et al., Chem. Phys. Lett. 439, 105 (2007).

${ }^{55}$ D. A. Weitz, S. Garoff, J. I. Gersten, and A. Nitzan, J. Chem. Phys. 78, 5324 (1983).

${ }^{56}$ P. K. Aravind and H. Metiu, Chem. Phys. Lett. 74, 301 (1980).

${ }^{57}$ K. Sokolov, G. Chumanov, and T. M. Cotton, Anal. Chem. 70, 3898 (1998).

${ }^{58}$ S. Hayashi, Surf. Sci. 158, 229 (1985).

${ }^{59}$ S. Garoff, D. A. Weitz, M. S. Alvarez, and J. I. Gersten, J. Chem. Phys. 81, 5189 (1984).

${ }^{60}$ P. K. Aravind and H. Metiu, Surf. Sci. 124, 506 (1983).

${ }^{61}$ A. Kinkhabwala, Z. F. Yu, S. H. Fan, Y. Avlasevich, K. Mullen, and W. E. Moerner, Nat. Photonics 3, 654 (2009).

${ }^{62}$ I. Kim, S. L. Bender, J. Hranisavljevic, L. M. Utschig, L. B. Huang, G. P. Wiederrecht, and D. M. Tiede, Nano Lett. 11, 3091 (2011).

${ }^{63}$ S. Kühn, U. Hakanson, L. Rogobete, and V. Sandoghdar, Phys. Rev. Lett. 97, 017402 (2006).

${ }^{64}$ H. Fischer and O. J. F. Martin, Opt. Express 16, 9144 (2008).

${ }^{65}$ R. Gill and E. C. Le Ru, Phys. Chem. Chem. Phys. 13, 16366 (2011).

${ }^{66}$ H. Cang, A. Labno, C. G. Lu, X. B. Yin, M. Liu, C. Gladden, Y. M. Liu, and $X$. Zhang, Nature (London) 469, 385-388 (2011).

${ }^{67}$ K. E. Sapsford, L. Berti, and I. L. Medintz, Angew. Chem., Int. Ed. 45, 4562 (2006). 